\title{
Salivary aldehyde dehydrogenase - temporal and population variability, correlations with drinking and smoking habits and activity towards aldehydes contained in food
}

\author{
Joanna Giebułtowicz¹, Marta Dziadek1, Piotr Wroczyński', Katarzyna Woźnicka², \\ Barbara Wojno², Monika Pietrzak² and Jacek Wierzchowski2® \\ 1Department of Bioanalysis and Drugs Analysis, Faculty of Pharmacy, Medical University of Warsaw, Warszawa, Poland; ${ }^{2}$ Department \\ of Biophysics, Faculty of Food Sciences, University of Warmia and Mazury, Olsztyn, Poland
}

Fluorimetric method based on oxidation of the fluorogenic 6-methoxy-2-naphthaldehyde was applied to evaluate temporal and population variability of the specific activity of salivary aldehyde dehydrogenase (ALDH) and the degree of its inactivation in healthy human population. Analyzed was also its dependence on drinking and smoking habits, coffee consumption, and its sensitivity to $\mathrm{N}$-acetylcysteine. Both the specific activity of salivary ALDH and the degree of its inactivation were highly variable during the day, with the highest activities recorded in the morning hours. The activities were also highly variable both intra- and interpersonally, and negatively correlated with age, and this correlation was stronger for the subgroup of volunteers declaring abstinence from alcohol and tobacco. Moderately positive correlations of salivary ALDH specific activity with alcohol consumption and tobacco smoking were also recorded $\left(r_{s} \sim 0.27\right.$; $p=0.004$ and $r_{\mathrm{s}}=0.30 ; p=0.001$, respectively). Moderate coffee consumption correlated positively with the inactivation of salivary ALDH, particularly in the subgroup of non-drinking and non-smoking volunteers. It was found that mechanical stimulation of the saliva flow increases the specific activity of salivary ALDH. The specific activity of the salivary ALDH was strongly and positively correlated with that of superoxide dismutase, and somewhat less with salivary peroxidase. The antioxidant-containing drug $\mathrm{N}$-acetylcysteine increased activity of salivary ALDH presumably by preventing its inactivation in the oral cavity. Some food-related aldehydes, mainly cinnamic aldehyde and anisaldehyde, were excellent substrates of the salivary ALDH3A1 enzyme, while alkenals, particularly those with short chain, were characterized by lower affinity towards this enzyme but high catalytic constants. The protective role of salivary ALDH against aldehydes in food and those found in the cigarette smoke is discussed, as well as its participation in diminishing the effects of alcohol- and smoking-related oxidative stress.

Keywords: aldehydes, aldehyde dehydrogenase, fluorescence, saliva, salivary peroxidase, superoxide dismutase, nutrition safety

Received: 12 April, 2010; revised: 01 August, 2010; accepted: 03 September, 2010; available on-line: 02 October, 2010

\section{INTRODUCTION}

Aldehydes are chemically reactive substances frequently encountered in the food, either as natural ingredients (citral, anisaldehyde, vanillin, cinnamic aldehyde and so on), products of food processing (e.g., pyrolysis), or as flavoring additives (Feron et al., 1991; Adams et al., 2007). Other potentially dangerous aldehydes which may be found in food are those generated during lipid peroxidation (Feron et al., 1991; Ellis, 2007), among these the most toxic 4-hydroxy-2-nonenal (Casetta et al., 2005; Ellis, 2007) and 4-oxo-hexenal (Kasai \& Kawai, 2008). Although some aldehydes are regarded as nontoxic to humans, at least in low concentrations (Adams et al., 1997; 2005; 2007), others, including acetaldehyde, are suspected in the pathogeny of cancer and other diseases (Seitz et al., 2001; Salaspuro, 2003; 2009; Casetta et al., 2005; Gowder \& Devaraj, 2010). In view of the high chemical reactivity of aldehydes, detailed analysis on their metabolism seems necessary to fully recognize potential dangers of food-related aldehydes.

Human salivary aldehyde dehydrogenase (ALDH, EC 1.2.1.5) is recognized, together with other enzymes, like glutathione $S$-transferases, as the first barrier against toxic aldehydes contained in the food or produced during lipid peroxidation (Sreerama et al., 1995; Sladek, 2004; Vasiliou et al., 2004). The salivary ALDH has been identified as a single ALDH3A1 isoenzyme (Dyck, 1995; Sreerama et al., 1995; Esser et al., 2008) of dimeric structure and broad substrate specificity (Pappa et al., 2003b; Wroczynski \& Wierzchowski, 2000; Giebułtowicz et al., 2009), albeit almost inactive towards acetaldehyde. An identical ALDH isoenzyme has been found to be highly active in the cytosol of stomach and lungs and especially in the cornea (Sladek, 2004; Vasiliou et al., 2004; Pappa et al., 2003a; 2003b; Lassen et al., 2008). A similar isoenzyme is frequently over-expressed in tumors, including those of salivary glands (Sreerama \& Sladek, 1994; 1996; Sladek, 2004). Of interest is also the activity of this enzyme against cyclophosphamide metabolites produced during cancer chemotherapy, closely correlated with cellular resistance to this drug (Sladek, 1999; Townsend et al., 2001; Sladek et al., 2002; Moreb et al., 2007).

The kinetic properties of the ALDH3A1 isoenzyme have been determined for a number of aldehydes both with $\mathrm{NAD}^{+}$and $\mathrm{NADP}^{+}$as co-substrates. Among the best substrates, both in terms of $K_{\mathrm{m}}$ and $V_{\max } / K_{\mathrm{m}}$, are cinnamic aldehyde, benzaldehyde and anisaldehyde, all of them of aromatic character (Giebułtowicz et al., 2009),

e-mail: jacek.wie@uwm.edu.pl

Abbreviations: ALDH, aldehyde dehydrogenase; ALDH3A1, the $3 A 1$ isoenzyme of the latter; DTT, dithiothreitol; GSH, glutathione; NBT, Nitro Blue Tetrazolium; SOD, superoxide dismutase; SPO, salivary peroxidase 
while long-chain alkenals exhibit somewhat lower affinity for the enzyme. The potentially mutagenic polyaromatic aldehydes exhibit especially low $K_{\mathrm{m}}$ values, well below $1 \mu \mathrm{M}$, and high catalytic constants (Wroczyński \& Wierzchowski, 2000; Glatt et al., 2008, Giebultowicz et al., 2009). The preferred co-substrate is $\mathrm{NAD}^{+}$, with the $K_{\mathrm{m}}$ in low micromolar range (Sladek, 2004; Wierzchowski et al., 2008). These data are in agreement with the hypothesis of an important role of salivary ALDH in aldehyde detoxication in the gastrointestinal tract.

To date, the only large-scale population study of the salivary ALDH activity is that of Sreerama et al. (1995) who used a spectrophotometric assay based on the detection of NADH formation during the enzymatic oxidation of benzaldehyde with $\mathrm{NAD}^{+}$. This paper demonstrated high population variability of the enzyme, its correlation with other detoxifying enzymes, and possibility of its induction by extensive consumption of some foodstuffs, like coffee or broccoli. Presently, activities of salivary ALDH can be measured specifically using a much more sensitive fluorimetric method based on enzymatic oxidation of fluorogenic naphthaldehydes like 6-methoxy-2-naphthaldehyde or 6-dimethylamino-2-naphthaldehyde (Wierzchowski et al., 1997; 2008; Wroczynski \& Wierzchowski, 2000; Giebułtowicz et al., 2009). This allowed us to perform a population study on the salivary ALDH activity in a healthy human population as well as in groups of patients suffering from various pathological states of the oral cavity (to be reported elsewhere).

In our previous papers (Wroczynski et al., 2004; Wierzchowski et al., 2008; Bogucka et al., 2009) we have shown that salivary ALDH undergoes reversible inactivation, presumably by air, already in the oral cavity. Inactivation of the salivary ALDH can be prevented in vitro by addition of $1 \mathrm{mM}$ glutathione, while treatment by dithiols like dithiothreitol or dithioerythritol leads to rapid and complete reactivation of the enzyme. The degree of ALDH inactivation in saliva can be therefore determined as the ratio of activities measured in the presence of glutathione (GSH) and dithiothreitol (DT'T), and preliminary studies have revealed that it can vary between zero and $>90 \%$ (Wroczynski et al., 2004; Wierzchowski et al., 2008; Bogucka et al., 2009). Some drugs are able to reverse (or prevent) salivary ALDH oxidation, at least in the short term (Bogucka et al., 2009). Identification of factors affecting both the activity and degree of inactivation of ALDH in saliva seems to be important for the study of aldehyde detoxication and, consequently, for nutrition safety.

The aim of the present work is an evaluation of population and temporal variability of the specific activity of salivary ALDH and its degree of inactivation, as well as preliminary examination of their relation to drinking and smoking habits among healthy human individuals. Addressed is also the sensitivity of salivary ALDH to some antioxidant substances used as drugs, notably $\mathrm{N}$-acetylcysteine, and the correlation of ALDH activity in saliva with those of salivary antioxidant enzymes, particularly superoxide dismutase and peroxidase.

\section{MATERIALS AND METHODS}

Enzymes and chemicals. Recombinant human ALDH3A1 was prepared and purified as described previously (Giebultowicz et al., 2009). Superoxide dismutase and xanthine oxidase were purchased from Sigma. Aldehydes and the corresponding carboxylic acids were purchased from Aldrich and re-crystallized if necessary. $\mathrm{NAD}^{+}, \mathrm{NADH}, \mathrm{N}$-acetylcysteine (99\%), 2,7-dichlorofluorescein, leuko-2,7-diacetylochlorofluorescin, xanthine, and NBT (nitro blue tetrazolium) were from Sigma. All other chemicals were of analytical grade, and water was filtered through the Millipore ultrafiltration system. Buffers were devoid of any fluorescent impurities.

Apparatus. A Cary 219 and Nicolet Evolution 300 spectrophotometers were used to perform spectral and kinetic study of enzyme activities. Fluorimetric measurements were done using Pekin-Elmer LS-50 or Shimadzu 5001 spectrofluorimeters. Absolute reaction rates were calculated using an internal standard (purified reaction product) at concentrations $1-2 \mu \mathrm{M}$.

Saliva collection. Saliva samples were collected directly to test tubes containing $50 \mathrm{mM}$ potassium phosphate, pH 7.3, $1 \mathrm{mM}$ GSH and $1 \mathrm{mM}$ EDTA. Saliva was diluted approximately $1: 1$, vigorously mixed, then centrifuged for about $10 \mathrm{~min}$ at $4000 \mathrm{rev} . / \mathrm{min}$, and the supernatant gently collected and stored in ice. Protein content in the supernatant was measured using Bradford reagent (Sigma). We found that the ALDH activity is stable for several hours in these conditions (Wierzchowski et al., 2008).

Saliva donation was preceded by filling out an anonymous interview, indicating age, sex, intake of drugs, alcohol and coffee as well as cigarette smoking during the past week.

Enzyme activities. Salivary ALDH activity was measured fluorimetrically, using 6-methoxy-2-naphthaldehyde $(5 \mu \mathrm{M})$ as the fluorogenic substrate, in the presence of $100 \mu \mathrm{M} \mathrm{NAD}^{+}$and either $0.5 \mathrm{mM} \mathrm{DT}^{\prime} \mathrm{T}$ or $1 \mathrm{mM} \mathrm{GSH}$, as described earlier (Wierzchowski et al., 1997; Wroczynski et al., 2004; Bogucka et al., 2009). The final dilution of the saliva sample in the cuvette was about $1: 40$. Fluorescence increase $(\mathrm{dF} / \mathrm{dt})$ of the produced 6-methoxy-2-naphthoate was recorded at $360 \mathrm{~nm}$, with excitation at $315 \mathrm{~nm}$, and after 6-10 min of reaction an aliquot of 6-methoxy2-naphthoic acid (Sigma) was added as an internal standard (final concentration about $1.5 \mu \mathrm{M}$ ). Absolute reaction rates were calculated according to the formula:

$v=\frac{d F}{d t} \frac{C_{s t}}{F_{s t}}$

where $C_{s t}$ and $F_{s t}$ are concentration (in $\mu \mathrm{M}$ ) and fluorescence of the internal standard, respectively. Specific activities were calculated as rate/protein content, multiplied by sample dilution. We estimate the standard error of this method to be $<5 \%$ for reaction rates and $7 \%-8 \%$ for specific activities (Wierzchowski et al., 1997; 2008).

One enzyme unit is defined as the amount oxidizing 1 micromole of 6-methoxy-2-naphthaldehyde per minute in the presence of $100 \mu \mathrm{M} \mathrm{NAD}{ }^{+}$at $25^{\circ} \mathrm{C}$. This unit is approximately twice as large as the commonly used benzaldehyde unit (Wierzchowski et al., 1997; Giebułtowicz et al., 2009).

The degree of salivary ALDH reversible inactivation (see above) was calculated as

$I=1-\frac{V_{G S H}}{V_{D T T}}$

where $V_{G S H}$ and $V_{D T T}$ are reaction rates determined in the presence of $1 \mathrm{mM} \mathrm{GSH}$ and $0.5 \mathrm{mM}$ DT'T, respectively. 
Kinetic examination of other ALDH substrates was performed using standard spectrophotometric procedures, with $\mathrm{NAD}^{+}$concentrations elevated to $300 \mu \mathrm{M}$, and occasionally checked fluorimetrically by monitoring fluorescence of NADH at $460 \mathrm{~nm}$.

The activity of salivary superoxide dismutase (SOD) was determined using a modified Beauchamp method (Beauchamp \& Fridovich, 1971). The reaction mixture $(1 \mathrm{~mL})$ contained $50 \mathrm{mM}$ sodium carbonate buffer, $\mathrm{pH}$ 10.2 , saturated by air at $25^{\circ} \mathrm{C}, 120 \mu \mathrm{M}$ EDTA, $120 \mu \mathrm{M}$ xanthine, $30 \mu \mathrm{M}$ NBT and $0.06 \mathrm{mg} / \mathrm{mL}$ BSA. Xanthine oxidase $(40 \mu \mathrm{L}$ of a stock solution with an activity of 1.6 $\mathrm{U} / \mathrm{mL}$ ) was added to start the reaction. Formation of diformazan was recorded spectrophotometrically at 560 $\mathrm{nm}$ first without saliva for $7 \mathrm{~min}$, and for the next $7 \mathrm{~min}$ with $100 \mu \mathrm{L}$ of the saliva supernatant added. The activity of SOD was calculated using a standard curve prepared with superoxide dismutase from bovine erythrocytes.

Salivary peroxidase (SPO) activity was determined fluorimetrically using the method described by Proctor and Chan (1994), and utilizing enzymatic oxidation of the fluorogenic leuko-2,7-dichlorodichlorofluorescin $(0.2 \mu \mathrm{M})$ to 2,7-dichlorofluorescein in the presence of $0.18 \mathrm{mM}$ $\mathrm{H}_{2} \mathrm{O}_{2}$ and $1 \mathrm{mM} \mathrm{KSCN}$ in $67 \mathrm{mM}$ potassium phosphate buffer, $\mathrm{pH}$ 6. A typical saliva dilution in the cuvette was $1: 150$. The reaction was performed at $25^{\circ} \mathrm{C}$. The method was modified by measuring the fluorescence slopes, $\mathrm{dF} / \mathrm{dt}$, rather than fluorescence increase $(\Delta \mathrm{F})$, and using purified 2,7-dichlorofluoresceine as an internal standard to determine absolute reaction rates. Fluorescence was recorded at $530 \mathrm{~nm}$ with excitation $488 \mathrm{~nm}$, and activity calculated using formula (1).

Statistical analysis. Normal distribution and homogeneity of variances of the data was checked with the Shapiro-Wilk test and the Leven's test, respectively. In case of a lack of normal distribution or homogeneity of variances $(p<0.05)$, subsequent statistical evaluation of significance was performed by Mann-Whitney U test (for independent samples) or by Wilcoxon test (for dependent samples). Otherwise, paired or unpaired Student's $t$-test was used for dependent and independent samples, respectively.

The correlation between parameter levels was analyzed with Spearman's rank correlation test, and the results expressed in terms of $r_{\mathrm{s}}$ (correlation coefficient) and $p$, indicating significance (tests showing $p<0.05$ were considered significant).

\section{RESULTS AND DISCUSSION}

Temporal variability of specific activity of salivary ALDH and of its inactivation degree

The human saliva content is known to vary with time due to various processes of saliva stimulation and other factors. It was therefore necessary to obtain information on the daily variability of salivary ALDH activity in order to establish proper conditions for a broader statistical evaluation of this enzyme.

In a preliminary experiment, the specific activity of salivary ALDH was measured using the fluorimetric method in four healthy, non-smoking volunteers throughout the day times, from 8:30 AM to 6 PM. For each sample the activity was determined twice, first in the presence of $1 \mathrm{mM}$ glutathione, and then with $0.5 \mathrm{mM}$ dithiothreitol, the latter results shown in Fig. 1 (upper panel). It is evident that the recorded ALDH activities vary with time of collection in a fairly consistent man- ner. The highest specific activities were recorded during the morning hours (about $9 \mathrm{AM}$ ), and decreased rapidly later. At the same time, evaluation of the enzyme inactivation (see Methods) shown that in the morning hours it was inactivated to a much lower extent (45-65\%) than in the afternoon, when the inactivation increased to 80 95\% (see Fig. 1, lower panel).

A similar experiment was subsequently conducted on 14 healthy volunteers, aged 20-60, with saliva collected trice a day, at 9 AM, 2 PM and 6 PM, but gave somewhat different results: the average activity determined in the presence of DTT decreased at 2 PM relative to 9 AM, by about $64 \%$, and remained almost unchanged at 6 PM, with high coefficients of variations $(>100 \%)$. The average ALDH inactivation degree in this group was $72 \%$ in the morning, to diminish later to $53 \%$ (2 PM) and then rise to $57 \%(6 \mathrm{PM})$. Although these data are not identical with those presented in Fig. 1, they nevertheless confirm the high daily variability of salivary ALDH specific activity and show that the inactivation degree of this enzyme is also variable. For this reason we decided to perform further studies of the salivary ALDH activity at a fixed collection time, between 8:30 and 10:00 AM.

The long term intra-individual variation of the salivary ALDH activity and inactivation degree was examined for the period of 3.5 months for four healthy volunteers, with saliva collected during morning hours (in most cases before the first meal, 8:30 to 9:30 AM), with results shown in Fig. 2. The diet was not controlled. There was a rather high variability in both the specific activity and degree of inactivation, with coefficients of variation
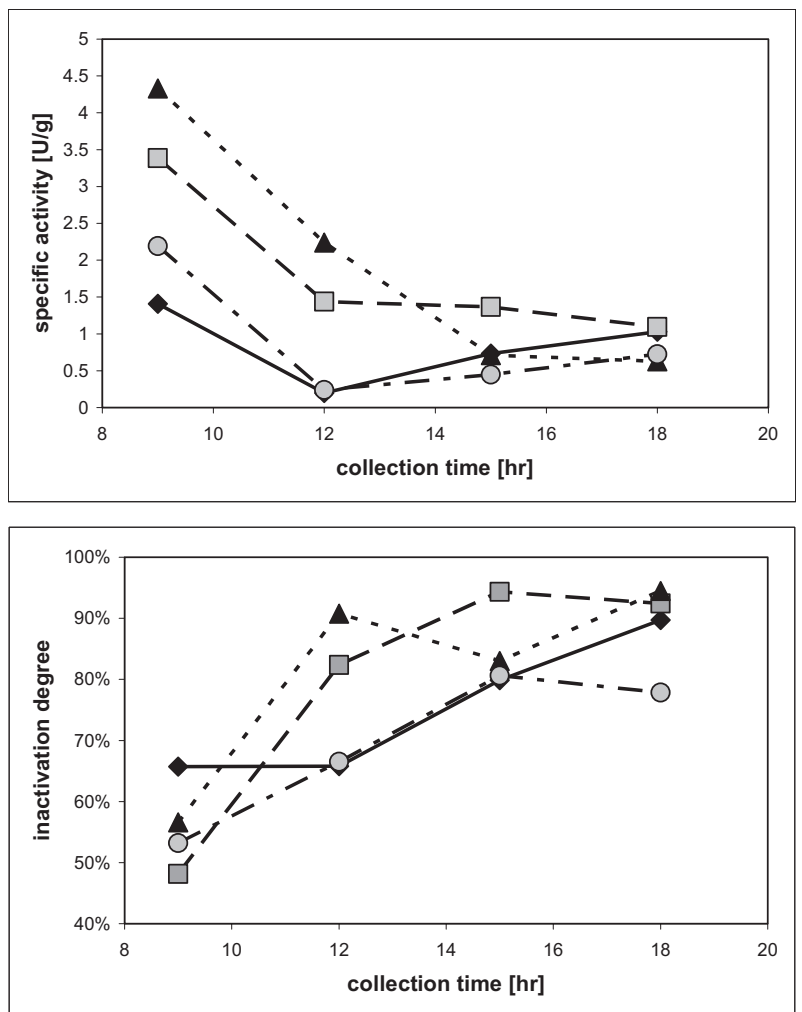

Figure 1. Daily evolution of salivary ALDH specific activity for four healthy, non-smoking volunteers

Activity was determined in the presence of $0.5 \mathrm{mM}$ DTT (upper panel) and degree of its inactivation calculated as explained in Methods (lower panel). 

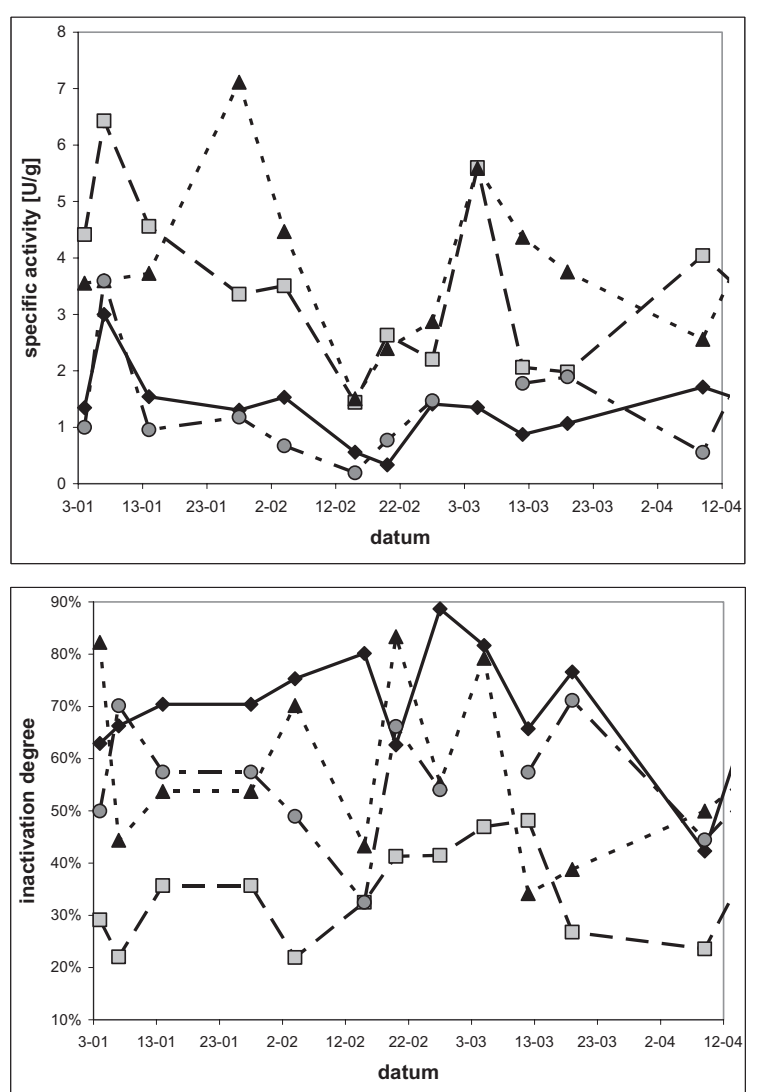

Figure 2. Long-term temporal evolution of salivary ALDH specific activity and degree of its inactivation recorded for four healthy, non-smoking volunteers for four months

Activity was determined in the presence of $0.5 \mathrm{mM}$ DTT (upper panel) and degree of its inactivation calculated as explained in Methods (lower panel). All saliva samples were collected between 8:30 to $9: 30$ AM.

(C.V.) for individual average ALDH activities from $42 \%$ to $70 \%$, and for the inactivation degrees from $20 \%$ to $29 \%$. These C.V. values are nevertheless markedly lower than those measured in the population study (see Table 1), and the recorded differences between individuals were in some cases statistically highly significant, based on a paired $t$-test $(p<0.001)$. This refers both to the "actual" and "total" activities (that is, those recorded in the presence of $1 \mathrm{mM} \mathrm{GSH}$ and $0.5 \mathrm{mM}$ DTT, respectively), and, additionally, to the degree of enzyme reversible inactivation (see Fig. 2). These data confirm that the human population is markedly diversified in terms of the salivary ALDH activity, and this diversification between individuals may persist over a fairly long time.

\section{Salivary ALDH in healthy human population}

The specific activity of salivary ALDH was investigated in two groups of volunteers: one from the Warsaw area $(\mathrm{N}=72)$, the second from less polluted area in the Varmia lake region $(\mathrm{N}=40)$. Individuals who suffered from metabolic diseases were excluded from analysis (based on the interview). All samples were collected between 8:30 AM and 10:00 AM, and an anonymous interview was obtained from each volunteer indicating their age, sex, health status, administered medicines and information related to drinking and smoking (see below). The results, analyzed selectively for each group, are presented in Table 1. Since the differences between the Varmia and Warsaw groups were not statistically significant ( $p>0.05$, based on the Mann-Whitney $U$ test), Table 1 includes also statistics for the pooled group.

Some statistically significant differences between the male and female sub-groups (Table 2) are probably related to differences in drinking and smoking habits between these groups (see below).

Statistical analysis indicated a moderate but significant negative correlation of the age of volunteers and the salivary ALDH activities as determined either in the presence of DTT $\left(r_{s}=-0.19, p=0.042\right)$ or GSH $\left(r_{\mathrm{s}}=-0.19\right.$, $p=0.041)$. This correlation was somewhat higher in the subgroup declaring complete alcohol and cigarette smoking abstinence $\left(r_{\mathrm{s}}=-0.43, p=0.012\right.$; and $r_{\mathrm{s}}=-0.40$, $p=0.018$ for activities measured in the presence of GSH and DTT, respectively; $\mathrm{n}=34$ ). No significant correlation between age and the ALDH inactivation degree was found $(p>0.1)$, except in the Varmia subgroup, where we found a modest relationship between these parameters $\left(\mathrm{N}=40 ; r_{\mathrm{s}}=0.30, p=0.05\right)$.

The obtained activity data (average about $3.7 \mathrm{U} / \mathrm{g}$, and high coefficients of variations) are comparable to those presented by Sreerama et al. (1995), who used benzaldehyde as the preferred substrate, bearing in mind that our units are 2-fold larger than those used by Sreerama (see Methods). Since the average protein content in the saliva is 4-6 g/L (Esser et al., 2008), simple recalculation gives total ALDH activity in this fluid close to 15-20 $\mathrm{U} / \mathrm{L}$, which is several-fold higher than the ALDH level in whole blood, as determined by analogous fluorimetric method (about $4 \mathrm{U} / \mathrm{L}$; see Wierzchowski et al., 1995). This comparison strongly suggests an important role of the salivary ALDH in the protection against toxic aldehydes.

\section{Influence of drinking and smoking habits}

Alcohol consumption and tobacco smoking as well as coffee consumption by volunteers during the week pre-

Table 1. Salivary ALDH specific activity, and ALDH inactivation degree, determined in two groups of healthy subjects: one from Warsaw and second from Varmia region Pooled data are given for comparison.

\begin{tabular}{|c|c|c|c|c|c|c|}
\hline Group (n) & Warsaw g & oup (72) & Varmia $\mathrm{g}$ & up (40) & Pooled c & ta $(112)$ \\
\hline \multirow[t]{2}{*}{ Age } & \multicolumn{2}{|c|}{$19-85$} & \multicolumn{2}{|c|}{$19-62$} & \multicolumn{2}{|c|}{$19-85$} \\
\hline & Sp. Activitya $[\mathrm{U} / \mathrm{g}]$ & Inactivation ${ }^{\mathrm{b}}[\%]$ & Sp. Activitya [U/g] & Inactivation ${ }^{b}[\%]$ & Sp. Activitya $[\mathrm{U} / \mathrm{g}]$ & Inactivation ${ }^{\mathrm{b}}[\%]$ \\
\hline Average & 3.52 & 63 & 3.95 & 61 & 3.67 & 62 \\
\hline Median & 2.53 & 72 & 2.56 & 64 & 2.53 & 67 \\
\hline Range & $0.14-20.8$ & $1-97$ & $0.63-13.57$ & 4-96 & $0.14-20.8$ & $1-97$ \\
\hline C.V. & $107 \%$ & $43 \%$ & $85 \%$ & $41 \%$ & $98 \%$ & $42 \%$ \\
\hline
\end{tabular}

aTotal ALDH activity, determined in the presence of $0.5 \mathrm{mM} \mathrm{DTT;}{ }^{\mathrm{b}} \mathrm{ALDH}$ inactivation degree, calculated as described in Methods; cDifferences between Warsaw and Varmia groups are not statistically significant. 
Table 2. Salivary ALDH activity, and its inactivation degree (see Methods) in healthy males and females

Statistically significant differences are indicated by asterisk $(*)$.

\begin{tabular}{lcccc}
\hline \multicolumn{2}{c}{ group } & \multicolumn{2}{c}{ Male group 50 } & \multicolumn{2}{c}{ Female group 62} \\
\hline age & \multicolumn{2}{c}{$20-75$} & \multicolumn{2}{c}{$19-85$} \\
\hline & Sp. Activity [U/g] & Inactivation [\%] & Sp. Activity [U/g] & Inactivation [\%] \\
\hline Average & $4.43^{*}$ & 65 & $3.06^{*}$ & 61 \\
Median & $3.13^{*}$ & 67 & $2.15^{*}$ & 64 \\
Range & $0.14-20.8$ & $13-97$ & $0.27-16.2$ & $1-96$ \\
C.V. & $92 \%$ & $35 \%$ & $101 \%$ & $48 \%$ \\
\hline
\end{tabular}

ceding saliva collection were examined by anonymous interview.

Spearman's analysis indicated a moderate positive correlation between the declared alcohol consumption and the ALDH total activity (as determined in the presence of DT' $)$ in the pooled population $(r=0.27 ; p=0.004)$ and somewhat weaker, but still significant, with actual ALDH activity, as measured in the presence of GSH $\left(r_{\mathrm{s}}=0.19, p=0.049\right)$. The correlation of alcohol consumption and ALDH inactivation degree was not statistically significant.

The above result may seem somewhat surprising, since acetaldehyde is known to be a very poor substrate for the salivary ALDH (Sladek, 2004; Vasiliou et al., 2004), so this enzyme probably does not participate directly in the protection against acetaldehyde-related toxicity. It may nevertheless play an indirect role in eliminating the effects of the alcohol-induced oxidative stress, which is known to lead to generation of toxic long-chain aliphatic aldehydes (Das \& Vasudevan, 2007; Ellis, 2007). An alternative explanation is that other substances used preferably by alcohol drinkers may be involved in the observed induction of the salivary ALDH.

Cigarette smoking is known to generate several volatile aldehydes, like acrolein and crotonaldehyde, supposedly involved in the etiology of cancers of the oral cavity (Nagler \& Reznick, 2004; Hasnis et al., 2004). Many of these aldehydes are substrates for the salivary ALDH3A1 (Pappa et al., 2003b; Vasiliou, 2004; and this paper, below), so some influence of cigarette smoking on salivary ALDH activity may be expected.

We have indeed found a significant, positive correlation between the declared cigarette smoking during the week preceding examination and the ALDH activity, as determined in the presence of DTT $\left(r_{\mathrm{s}}=0.30 ; p=0.001\right)$ and GSH $\left(r_{\mathrm{s}}=0.29 ; p=0.002\right)$. There was no correlation with the degree of ALDH inactivation $(p>0.1)$. The correlation between ALDH total activity (as measured in the presence of DT'T) and declared cigarette smoking was much stronger in the group from the Varmia region ( $\left.\mathrm{n}=40, r_{\mathrm{s}}=0.56, p=0.0002\right)$ than in that from Warsaw ( $\left.\mathrm{n}=72, \mathrm{r}_{\mathrm{s}}=0.26, p=0.024\right)$, suggesting an important role of air pollutants other than cigarette smoke in the ALDH induction. In the male sub-groups the correlations were even stronger than in the pooled groups, according to Spearman's test $\left(r_{\mathrm{s}}=0.64, p=0.007\right.$; and $r_{\mathrm{s}}=0.42, p=0.012$, for the Varmia and Warsaw groups, respectively), while the female subgroups of Varmia and Warsaw did not show any significant correlations $(p>0.1)$, but these subgroups contained much less heavy smokers. The above results show that salivary ALDH is probably induced by cigarette smoking and therefore is likely to be a part of the defensive mechanism against aldehydes contained in smoke, and possibly in other air pollutants.

\section{Influence of coffee consumption on salivary ALDH}

Extensive coffee consumption has been shown to increase salivary ALDH activity (Sreerama et al., 1995). Surprisingly, in the pooled data we found no significant correlation between the declared coffee consumption and the salivary ALDH activity (not shown). However, in the subgroup of absolute nondrinkers and non-smokers (based on interview, $\mathrm{n}=34$ ), a significant negative correlation was found between actual ALDH activity (measured in the presence of GSH) and coffee consumption $\left(r_{\mathrm{s}}=-0.44\right.$; $p=0.009)$, together with a strongly positive correlation between coffee consumption and the degree of ALDH inactivation $\left(r_{\mathrm{s}}=0.58, p=0.0003\right)$. In a broader group including moderate drinkers (less than $40 \mathrm{~g}$ alcohol and one pack of cigarettes per week, $n=48$ ) the latter correlation was still present, but less pronounced $\left(r_{\mathrm{s}}=0.40\right.$, $p=0.005)$. The correlation between coffee drinking and total ALDH activity (determined in the presence of DTT) in these two subgroups was not statistically significant $(p>0.05$, not shown).

The above data do not confirm the observations reported by the Sládek's group (Sreerama et al., 1995) of a strong induction of salivary ALDH by coffee consumption. This may be probably explained by the fact that in our group of volunteers the number of individuals consuming more than three cups of coffee per day was very small (6 persons), while the Sreerama's observations refer to consumption of one liter of coffee per day or more. It is interesting to note in this context that salivary ALDH is directly inhibited by caffeine (Wierzchowski et al., 2008), so the observed induction may be a part of the mechanism improving defensive abilities of human saliva in those cases when ALDH activity is markedly lowered.

Our results indicate also that moderate coffee drinking leads to inactivation of the salivary ALDH, especially among the non-drinkers and non-smokers. A possible mechanism of this phenomenon may be an increased activity of xanthine oxidase, resulting in the generation of superoxide radicals, acting directly or indirectly (e.g., via depletion of GSH) on ALDH.

\section{Resting vs. stimulated saliva}

Activities of the salivary enzymes vary naturally as an effect of the stimulation of the saliva flux. We examined whether mechanical stimulation of saliva flux by chewing Parafilm ${ }^{\circledR}$ foil affects salivary ALDH3A1 specific activity (that is, related to total protein) and degree of its inactivation. Volunteers $(n=23)$ were asked to donate saliva samples prior and during extensive Parafilm ${ }^{\circledR}$ chewing for approx. $5 \mathrm{~min}$. The results are presented in Table 3.

The observed increase in ALDH3A1 specific activity after stimulation of saliva flux (Table 3 ) is statistically significant. The Wilcoxon matched-pairs signed-ranks test gave $p=0.013$ and 0.0054 for the increase of the activities measured in the presence of GSH and DT'T, respectively. There was no significant change in the ALDH inactivation degree $(p>0.1)$. This again suggests that salivary ALDH may be important as a protective 
Table 3. Influence of stimulation of saliva flux on ALDH specific activity and its inactivation degree

The group included 23 persons (14 females), aged $18-70$. Statistically significant changes $(p<0.05)$ are indicated by asterisk $(*)$.

\begin{tabular}{|c|c|c|c|c|c|c|}
\hline \multirow[t]{2}{*}{ Group } & \multicolumn{3}{|c|}{ Prior to stimulation } & \multicolumn{3}{|c|}{ During stimulation ${ }^{a}$} \\
\hline & $\begin{array}{l}\text { Sp. Activity }(G S H)^{b} \\
{[U / g]}\end{array}$ & $\begin{array}{l}\text { Sp. Activity (DTT)c } \\
{[\mathrm{U} / \mathrm{g}]}\end{array}$ & $\begin{array}{l}\text { Inactivation } \\
\text { degreed }^{d}\end{array}$ & $\begin{array}{l}\text { Sp. Activity (GSH) } \\
{[\mathrm{U} / \mathrm{g}]}\end{array}$ & $\begin{array}{l}\text { Sp. Activity (DTT)c } \\
{[\mathrm{U} / \mathrm{g}]}\end{array}$ & $\begin{array}{l}\text { Inactivation } \\
\text { degreed }^{d}\end{array}$ \\
\hline Average & $0.99 *$ & $2.16^{*}$ & 0.58 & $1.87^{*}$ & $3.86^{*}$ & 0.49 \\
\hline Median & $1.15^{*}$ & $1.42^{*}$ & 0.63 & $2.02^{*}$ & $1.88^{*}$ & 0.47 \\
\hline C.V. & $116 \%$ & $89 \%$ & $35 \%$ & $108 \%$ & $95 \%$ & $51 \%$ \\
\hline
\end{tabular}

aSaliva flux was stimulated by chewing parafilm ${ }^{\circledR}$ foil for about 5 min; ${ }^{b}$ Actual ALDH activity, determined in the presence of 1 mM GSH; ${ }^{\mathrm{C} T o t a l}$ ALDH activity, determined in the presence of $0.5 \mathrm{mM} \mathrm{DTT}$; ${ }^{\mathrm{d} C a l c u l a t e d}$ using formula $\{2\}$ (see Methods).

enzyme during the consumption of chewable foodstuffs like raw vegetables or fruit, usually containing volatile aldehydes as natural flavors.

\section{Correlation of ALDH with salivary superoxide dismutase and oral peroxidase activities}

The salivary ALDH activity has been demonstrated to correlate with the activities of typical phase II detoxication enzymes like glutathione- $S$-transferases and DT-diaphorase (Sreerama et al., 1995). However, aldehyde elimination is considered also a part of the defensive mechanism against oxidative stress, since active oxygen species lead to aldehyde generation during lipid peroxidation (Nagler \& Reznick, 2004; Hasnis et al., 2004; Das \& Vasudevan, 2006; Ellis, 2007). Furthermore, the cellular regulation of ALDH3A1 expression seems to involve both xenobiotic-responsive elements (XRE) and electrophile-antioxidant responsive elements (ARE) (Sladek, 2004; Vasiliou et al., 2004; Ellis, 2007), suggesting dual role of this enzyme in maintaining cellular homeostasis. We therefore examined possible correlations of the salivary ALDH with two most important antioxidant activities of the saliva, that is superoxide dismutase (SOD) and salivary peroxidase (SPO; see Nagler et al., 2002; Inhalin et al., 2006; Ashby, 2008).

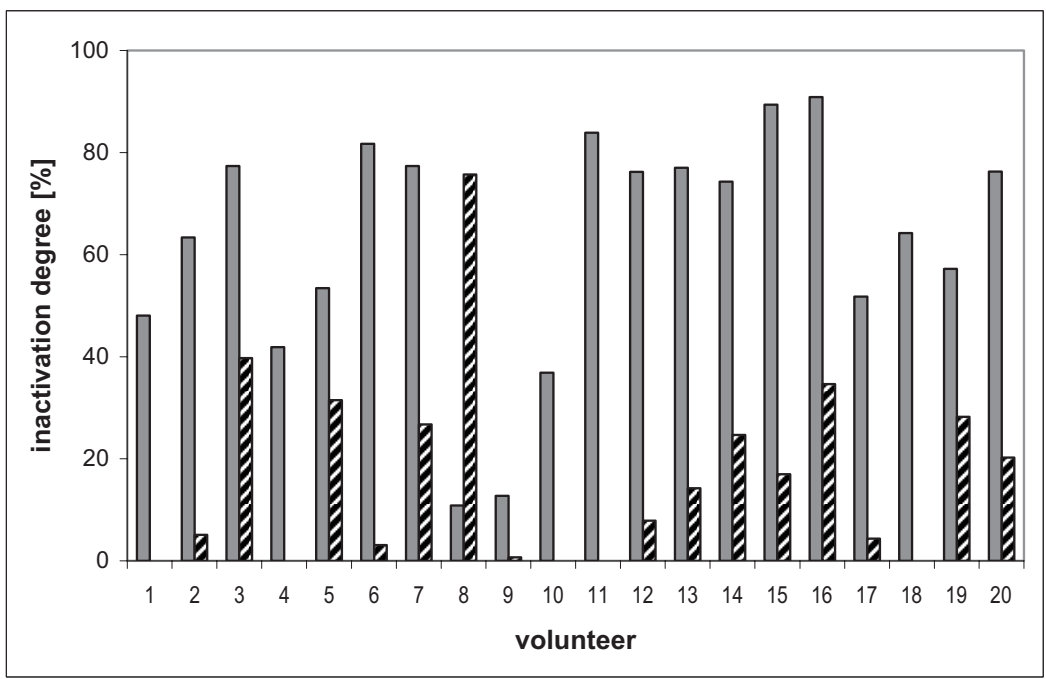

Figure 3. Effect of $\mathrm{N}$-acetylcysteine on the salivary ALDH inactivation

Salivary ALDH activity was assayed in 20 healthy volunteers first in the presence of 1 $\mathrm{mM}$ GSH and next with $0.5 \mathrm{mM}$ DTT, and inactivation degree calculated as described in Methods. Bars show ALDH inactivation prior to (filled bars) and $30 \mathrm{~min}$ after (dashed bars) washing mouth with $5 \mathrm{~g} / \mathrm{L}$ solution of $\mathrm{N}$-acetylcysteine in the presence of citric acid.
The activities of SOD and salivary peroxidase were measured in the saliva of 28 healthy volunteers (all from the Warsaw group) and correlations with salivary ALDH examined. We found a highly significant, strongly positive correlation between the activity of salivary superoxide dismutase and total ALDH activity (as determined in the presence of DTT) with $r_{\mathrm{s}}=0.57, p<0.00001$; and a significant positive correlation with actual ALDH (determined in the presence of GSH; $\left.r_{\mathrm{s}}=0.43, p=0.00002\right)$. There was apparently no significant correlation of the SOD activity and ALDH inactivation degree (not shown). We also found moderate correlations between salivary peroxidase activity and both actual ALDH (i.e., measured in the presence of GSH; $\left.r_{\mathrm{s}}=0.25, p=0.009\right)$ and ALDH inactivation degree $\left(r_{\mathrm{s}}=-0.25, p=0.007\right)$.

The foregoing data, although preliminary, suggest that salivary ALDH may be involved in the defense against lipid peroxidation-related toxicity.

\section{Effect of $\mathrm{N}$-acetylcysteine}

We have recently reported that Fluimucil ${ }^{\circledR}$, a drug containing $N$-acetylcysteine as the active substance, prevents inactivation of salivary ALDH (Bogucka et al., 2009), at least for a few hours after administration. To check if $N$-acetylcysteine is in fact the main factor activating salivary ALDH, we examined the ALDH inactivation degree in the saliva of 20 volunteers prior to and after washing mouth for $1 \mathrm{~min}$ with a $5 \mathrm{~g} / \mathrm{L}$ solution of $\mathrm{N}$-acetylcysteine mixed with citric acid $(1 \mathrm{~g} / \mathrm{L})$. Only one volunteer exhibited an increase in the degree of ALDH inactivation (that is, decrease in activity) after the washing, while for the remaining 19 the inactivation was markedly diminished, sometimes to virtually $0 \%$ (cf. Fig. 3). The average ALDH inactivation degree prior to washing was 0.62 (C.V. $38 \%$ ), and after the washing 0.17 (C.V. 115\%), a highly significant result according to the Wilcoxon test $(p=0.0009)$. In a control group ( $\mathrm{n}=5)$, washing the mouth with citric acid only, the respective numbers were 0.57 $(15 \%)$ and $0.45(48 \%)$. The latter number is significantly different from that for the main experimental group ( $p=0.013$ according to MannWhitney $U$ test). The total activity of ALDH, as measured in the presence of DT'T, was apparently not affected (not shown). 
Table 4. Kinetic parameters for enzymatic oxidation of selected food-related aldehydes by recombinant human ALDH3A1 If not indicated otherwise, parameters were determined in the presence of $300 \mu \mathrm{MNAD}^{+}$, at $25^{\circ} \mathrm{C}$ and $\mathrm{pH}$ 7.3.

\begin{tabular}{lll}
\hline Substrate & $K_{\mathrm{m}}[\mu \mathrm{M}]$ & $\mathrm{V}_{\max }$ [relative $]$ \\
\hline Benzaldehyde & $148^{\mathrm{a}}\left(200^{\mathrm{b}}\right)$ & 100 \\
Cinnamic aldehyde $^{\mathrm{a}}$ & 6 & 160 \\
Anisaldehyde $^{\mathrm{a}}$ & 19 & 73 \\
Vanillin (pH 7.3) $^{\mathrm{a}}$ & 152 & 6 \\
Vanillin (pH 6.3) & $70 \pm 20$ & $\sim 10$ \\
Methylvanillin & $\sim 130$ & $\sim 60$ \\
Furfural & $>1000$ & $\mathrm{nd}$ \\
Citral & 218 & $\sim 4$ \\
Crotonaldehyde (trans-2-butenal) & $\sim 1100$ & $\sim 40$ \\
trans-2-hexenal & $162\left(155^{\mathrm{b}}\right)$ & $72\left(\sim 7^{\mathrm{b}}\right)$ \\
trans-2-nonenal & $<100\left(12^{\mathrm{b}}\right)$ & $\sim 90\left(26^{\mathrm{b}}\right)$ \\
4-hydroxy-2-nonenal & $45^{\mathrm{b}}$ & $\sim 5^{\mathrm{b}}$
\end{tabular}

adata from Giebułtowicz et al. (2009), determined in the presence of $100 \mu \mathrm{M} \mathrm{NAD}^{+}$; ${ }^{\text {b data }}$ from Pappa et al. (2003b), determined with 0.5 $\mathrm{mM} \mathrm{NADP}+$ as a co-substrate.

Salivary ALDH undergoes reversible inactivation, presumably by air, as do some other ALDH isoenzymes, particularly ALDH1A1 (Vallari \& Pietruszko, 1982). In both cases reactivation is promoted by dithiols like DTT. It is also known that the main active metabolite of $N$-acetylcysteine is GSH (Atkuri et al., 2007), and this drug is used as an antidote in cases of cysteine/GSH deficiency. However, cysteine or GSH alone are not able to reactivate the oxidized ALDH3A1 (Wierzchowski et al., 2008; Bogucka et al., 2009). We think therefore that $N$-acetylcysteine does not directly reactivate salivary ALDH, but rather prevents its inactivation, presumably via the radical-scavenging activity in the oral cavity and/ or in salivary glands.

The above experiment is important because it demonstrates a possibility to increase salivary ALDH activity by a relatively simple and safe pharmacological treatment.

\section{Substrate specificity of salivary ALDH and recombinant ALDH3A1 towards food-related aldehydes}

We have previously reported that some food-related aldehydes, like cinnamic aldehyde or anisalehyde, are excellent substrates for the salivary ALDH, and the kinetic parameters for enzymatic oxidation of a number of aromatic aldehydes using saliva or recombinant ALDH3A1 are virtually identical (Giebułtowicz et al., 2009). We now extended this examination to selected aliphatic aldehydes, commonly used as food additives or found as natural food ingredients, using spectrophotometric and fluorimetric methods. The results are summarized in Table 4.

As pointed out previously (Pappa et al., 2003a; Giebułtowicz et al., 2009), recombinant ALDH3A1 prefers long-chain aliphatic and aromatic aldehydes as substrates, with poor activity towards acetaldehyde. It is evident from Table 4 that ALDH3A1 is an efficient catalyst of common food-related aldehydes, especially those of aromatic character: cinnamic aldehyde, anisaldehyde, and, to a lesser extent, benzaldehyde and vanillin. The latter compound (3-methoxy-4-hydroxybenzaldehyde) seems to be a somewhat better substrate at a more acidic $\mathrm{pH}$, where its phenolic proton is mostly undissociated $\left(\mathrm{p} K_{a}\right.$ about 7). In agreement with the foregoing, 4-methylvanillin is a good substrate as well (Table 4). Somewhat surprisingly, furfural, which is known to be rapidly eliminated from the human organism and essentially safe (Adams et al., 1997), was found to be a very poor substrate of ALDH3A1, possibly for steric reasons.

Alkenals are frequently used as food additives (Adams et al., 2007). As evident from Table 4, crotonaldehyde and trans-hexenal are characterized by catalytic constants comparable to that of benzaldehyde, but also by rather high $K_{\mathrm{m}}$ values, which for crotonaldehyde reach millimolar level. Citral, which is a mixture of two isomeric octadienals, exhibited relatively low catalytic constant, about $5 \%$ of that of benzaldehyde, and a high $K_{\mathrm{m}}$ (about $200 \mu \mathrm{M})$. It is therefore rather questionable whether salivary ALDH3A1 can effectively contribute to the metabolic elimination of aldehydes of non-aromatic character, especially short-chain ones like crotonaldehyde. It is, however, of interest that some of the most toxic aldehydes, like 4-hydroxy-2-nonenal, and the potentially mutagenic polyaromatic aldehydes exhibit high affinity towards the ALDH3A1 isoenzyme, with $K_{\mathrm{m}}$ values in the low micromolar or submicromolar region, and high catalytic constants (Pappa et al., 2003a; Glatt et al., 2008; Giebułtowicz et al., 2009). This again indicates an important role of this isoenzyme in detoxication of food- and pollution-related aldehydes.

\section{CONCLUDING REMARKS}

Oxidation of food-related aldehydes to the corresponding carboxylic acids is a fairly complex process, catalyzed by various detoxifying enzymes, among them those belonging to the aldehyde dehydrogenase superfamily (Adams, 1997). We have demonstrated that the only ALDH isoenzyme present in the human saliva, ALDH3A1, may be in fact important for detoxication of at least some of these compounds, and this process probably starts already in the oral cavity, thanks to the good catalytic properties of salivary ALDH3A1. We have also shown that the activity of this enzyme in the saliva (and, possibly, in the stomach) can be modified by various factors, including diet, administered drugs as well as drinking and smoking. All these factors should be taken into account in determination of the acceptable levels of various aldehydes in the food, especially those used as food additives.

\section{Acknowledgement}

This work was supported by the Ministry of Science and Higher Education (Poland), grant No. N312 035 $31 / 2169$.

We thank to Dr. Janusz Chmaj for discussions regarding statistical analysis.

\section{REFERENCES}

Adams TB, Doull J, Goodman JI, Munro IC, Newberne P, Portoghese PS, Smith RL, Wagner BM, Weil CS, Woods LA, Ford RA (1997) The FEMA GRAS assessment of furfural used as a flavour ingredient. Food Chem Toxicol 35: 739-751.

Adams TB, Cohen SM, Doull J, Feron VJ, Goodman JI, Marnett LJ, Munro IC, Portoghese PS, Smith RL, Waddell WJ, Wagner BM (2005) The FEMA GRAS assessment of phenethyl alcohol, aldehyde, acid, and related acetals and esters used as flavor ingredients. Food Chem Toxicol 43: 1179-1206.

Adams TB, Gavin CL, Tavlor SV, Waddell WJ, Cohen SM, Feron, VI, Goodman J, Rietjens IMCM, Marnett LJ, Portoghese PS, Smith RL 
(2007) The FEMA GRAS assessment of $\alpha, \beta$-unsaturated aldehydes and related substances used as flavor ingredients. Food Chem Toxicol 46: $2935-2967$.

Ashby MT (2008) Inorganic chemistry of defensive peroxidases in the human oral cavity. J Dent Res 87: 900-914.

Atkuri KR, Mantovani JJ, Herzenberg LA, Herzenberg (2007) N-acetylcysteine — a safe antidote for cysteine/glutathione deficiency. Curr Opin Pharmacol 7: 355-359.

Bogucka M, Giebułtowicz J, Zawada K, Wroczyński P, Wierzchowski J, Pietrzak M, Piekarczyk P, Romanowska K (2009) The oxidation status of ALDH3A1 in human saliva and its correlation with antioxidant capacity measured by ORAC method. Acta Pol Pharm Drug Res 66: 477-482.

Beauchamp C, Fridovich I (1971) Superoxide dismutase: Improved assays and an assay applicable to acrylamide gels. Anal Biochem 44: 276-287.

Casetta I, Govoni V, Granieri E (2005) Oxidative stress, antioxidants and neurodegenerative diseases. Curr Pharm Des 11: 2033-2052.

Das SK, Vasudevan DM (2007) Alcohol-induced oxidative stress. Life Sci 81: 177-187.

Dyck LE (1995) Polymorphism of a class 3 aldehyde dehydrogenase present in human saliva and in hair roots. Alcohol Clin Exp Res 19: $420-426$.

Ellis EM (2007) Reactive carbonyls and oxidative stress: Potential for therapeutic intervention. Pharmacol Therap 115: 13-24.

Esser D, Alvarez-Llamas G, de Vries M, Weening D, Vonk RJ, Roelofsen H (2008) Sample stability and protein composition of saliva: Implications for its use as a diagnostic fluid. Biomarker Insights 2008: 25-37.

Feron VJ, Til HP, de Vrijer F, Woutersen RA, Cassee FR, van Bladeren PJ (1991) Aldehydes: occurrence, carcinogenic potential, mechanism of action and risk assessment. Mutat Res 259: 363-385.

Giebułtowicz J, Wolinowska M, Sztybor A, Pietrzak M, Wroczynski P, Wierzchowski J (2009) Salivary aldehyde dehydrogenase: activity towards aromatic aldehydes and comparison with recombinant ALDH3A1. Molecules 14: 2363-2372.

Glatt H, Rost K, Frank H, Seidel A, Kollock R (2008) Detoxification of promutagenic aldehydes derived from methylpyrenes by human aldehyde dehydrogenases ALDH2 and ALDH3A1. Arch Biochem Biophys 477: 196-205.

Gowder S, Devaraj H (2010) A review of the nephrotoxicity of the food flavor cinnamaldehyde. Curr Bioact Compd 6: 106-117.

Hasnis E, Reznick AZ, Pollack S, Klein Y, Nagler RM (2004) Synergistic effect of cigarette smoke and saliva on lymphocytes - The mediatory role of volatile aldehydes and redox active iron and the possible implications for oral cancer. Int J Biochem Cell Biol 36: 826-839.

Ihalin R, Loimaranta V, Tenovuo J (2006) Origin, structure, and biological activities of peroxidases in human saliva. Arch Biochem Biophys 445: 261-268.

Kasai H, Kawai K (2008) 4-oxo hexenal, a mutagen formed by $\omega-3$ fat peroxidation. Mutat Res 659: 56-59.

Lassen N, Black WJ, Estey T, Vasiliou V (2008) The role of corneal crystallins in the cellular defense mechanisms against oxidative stress. Sem Cell Dev Biol 19: 100-112.

Moreb SJ, Muhoczy D, Ostmark B, Zucali JR (2007) RNAi-mediated knockdown of aldehyde dehydrogenase class-1A1 and class-3A1 is specific and reveals that each contributes equally to the resistance against 4-hydroperoxycyclophosphamide. Cancer Chemother Pharmacol 59: $127-136$.

Nagler RM, Klein I, Zarzhevsky N, Drigues N, Reznick AZ (2002) Characterization of the differentiated antioxidant profile of human saliva. Free Radic Biol Med 32: 268-277.

Nagler RM, Reznick AZ (2004) Cigarette smoke effects on salivary antioxidants and oral cancer - Novel concepts Isr Med Assoc J 6: 691-694.

Pappa A, Chen C, Koutalos Y, Townsend AJ, Vasiliou V (2003a). ALDH3A1 protects human corneal epithelial cells from ultraviolet- and 4-hydroxy-2-nonenal-induced oxidative damage. Free Radic Biol Med 34: 1178-1189.

Pappa A, Estey T, Manzer R, Brown D, Vasiliou V (2003b) Human aldehyde dehydrogenase 3A1 (ALDH3A1): Biochemical characterization and immunohistochemical localization in the cornea. Biochem J 376: 615-623.

Proctor GB, Chan K (1994) A fluorometric assay of peroxidase utilizing 2',7'-dichlorofluorescin with thiocyanate: application to the study of salivary secretion. J Biochem Biophys Methods 28: 329-336.

Salaspuro MP (2003) Alcohol consumption and cancer of the gastrointestinal tract. Best Pract Res Clin Gastroenterol 17: 679-694.

Salaspuro MP (2009) Acetaldehyde as a common denominator and cumulative carcinogen in digestive tract cancers. Scand J Gastroenterol 44: 912-925.

Seitz HK, Matsuzaki S, Yokoyama A, Homann N, Väkeväinen S, Xian Dong Wang (2001) Alcohol and cancer. Alcohol Clin Exp Res 25: 137S-143S.

Sládek NE (1999) Aldehyde dehydrogenase-mediated cellular relative insensitivity to the oxazaphosphorines. Curr Pharm Des 5: 607-625.

Sládek NE (2004) Human aldehyde dehydrogenases: Potential pathological, pharmacological, and toxicological impact. J Biochem Mol Toxicol 17: 7-23.

Sládek NE, Kollander R, Sreerama L, Kiang DT (2002) Cellular levels of aldehyde dehydrogenases (ALDH1A1 and ALDH3A1) as predictors of therapeutic responses to cyclophosphamide-based chemotherapy of breast cancer: A retrospective study. Cancer Chemother Pharmacol 49: 309-321.

Sreerama L, Sládek NE (1996) Over-expression of glutathione S-transferases, DT-diaphorase and an apparently tumour-specific cytosolic class-3 aldehyde dehydrogenase by warthin tumours and mucoepidermoid carcinomas of the human parotid gland. Arch Oral Biol 41: 597-605.

Sreerama L, Sládek NE (1994) Identification of a methylcholanthreneinduced aldehyde dehydrogenase in a human breast adenocarcinoma cell line exhibiting oxazaphosphorine-specific acquired resistance. Cancer Res 54: 2176-2185.

Sreerama L, Hedge MW, Sládek NE (1995) Identification of a class 3 aldehyde dehydrogenase in human saliva and increased levels of this enzyme, glutathione S-transferases, and DT-diaphorase in the saliva of subjects who continually ingest large quantities of coffee or broccoli. Clin Cancer Res 1: 1153-1165.

Townsend AJ, Leone-Kabler S, Haynes RL, Wu Y, Szweda L, Bunting KD (2001) Selective protection by stably transfected human ALDH3A1 (but not human ALDH1A1) against toxicity of aliphatic aldehydes in V79 cells. Chem Biol Interact 130-132: 261-273.

Vallari RC, Pietruszko R (1982) Human aldehyde dehydrogenase: Mechanism of inhibition by disulfiram. Science 216: 637-639.

Vasiliou V, Pappa A, Estey T (2004) Role of human aldehyde dehydrogenases in endobiotic and xenobiotic metabolism. Drug Metab Rev 36: 279-299.

Wierzchowski J, Interewicz E, Wroczynski P, Orlanska I (1995) Continuous fluorimetric assay for human aldehyde dehydrogenase and its application to blood analysis. Anal Chim Acta 319: 209-219.

Wierzchowski J, Wroczynski P, Laszuk K, Interewicz E (1997) Fluorimetric detection of aldehyde dehydrogenase activity in human blood, saliva and organ biopsies, and kinetic differentiation between class I and class III isozymes. Anal Biochem 245: 69-78.

Wierzchowski J, Pietrzak M, Szeląg M, Wroczyński P (2008) Salivary aldehyde dehydrogenase - reversible oxidation of the enzyme and its inhibition by caffeine, investigated using fluorimetric method. Arch Oral Biol 53: 423-428.

Wroczyński P, Wierzchowski J (2000) Aromatic aldehydes as fluorogenic indicators for human aldehyde dehydrogenases and oxidases. Analyst 125: 511-516.

Wroczyński P, Wierzchowski J, Rakowska A, Chimkowska M, Targoński J (2004) Aldehyde dehydrogenase in human saliva evaluation of its oxidation status. Acta Pol Pharm 61 (Suppl): 62-64. 